\title{
The role of Raf-1 kinase inhibitor protein in the regulation of pancreatic beta cell proliferation in mice
}

\author{
F. N. Pardo • J. Altirriba • M. Pradas-Juni • A. García • \\ U. Ahlgren • A. Barberà • J. C. Slebe • A. J. Yáñez • \\ R. Gomis • R. Gasa
}

Received: 19 March 2012 / Accepted: 27 July 2012 /Published online: 29 August 2012

(C) Springer-Verlag 2012

\begin{abstract}
Aims/hypothesis Manoeuvres aimed at increasing beta cell mass have been proposed as regenerative medicine strategies for diabetes treatment. Raf-1 kinase inhibitor protein 1 (RKIP1) is a common regulatory node of the mitogen-activated protein kinase (MAPK) and nuclear factor $\kappa \mathrm{B}(\mathrm{NF}-\mathrm{\kappa} \mathrm{B})$ pathways and therefore may be involved in regulation of beta cell homeostasis. The aim of this study was to investigate the involvement of RKIP1 in the control of beta cell mass and function. Methods Rkipl (also known as Pebpl) knockout (Rkip1 ${ }^{-1}$ ) mice were characterised in terms of pancreatic and glucose homeostasis, including morphological and functional analysis. Glucose tolerance and insulin sensitivity were examined, followed by assessment of glucose-induced insulin secretion
\end{abstract}

Electronic supplementary material The online version of this article (doi:10.1007/s00125-012-2696-9) contains peer-reviewed but unedited supplementary material, which is available to authorised users.

F. N. Pardo $\cdot$ J. Altirriba $\cdot$ M. Pradas-Juni $\cdot$ A. García $\cdot$

A. Barberà $\cdot$ R. Gomis $(\bowtie) \cdot$ R. Gasa $(\bowtie)$

Laboratory of Diabetes and Obesity,

IDIBAPS, Centre Esther Koplowitz,

5th Floor, Rosselló, 153,

08036 Barcelona, Spain

e-mail: rgomis@clinic.ub.es

e-mail: rgasa@clinic.ub.es

F. N. Pardo · J. Altirriba • M. Pradas-Juni • A. García • R. Gomis •

R. Gasa

Centro de Investigación Biomédica en Red de Diabetes y

Enfermedades Metabólicas Asociadas (CIBERDEM), Spain

URL: www.ciberdem.org/

F. N. Pardo • J. C. Slebe • A. J. Yáñez

Instituto de Bioquímica y Microbiología, Facultad de Ciencias,

Universidad Austral de Chile,

Valdivia, Chile in isolated islets and beta cell mass quantification through morphometry. Further characterisation included determination of endocrine and exocrine proliferation, apoptosis, MAPK activation and whole genome gene expression assays. Capacity to reverse a diabetic phenotype was assessed in adult Rkip1 ${ }^{-1-}$ mice after streptozotocin treatment.

Results Rkip1 $1^{-1}$ mice exhibit a moderately larger pancreas and increased beta cell mass and pancreatic insulin content, which correlate with an overall improvement in whole body glucose tolerance. This phenotype is established in young postnatal stages and involves enhanced cellular proliferation without significant alterations in cell death. Importantly, adult Rkip $1^{-/}$mice exhibit rapid reversal of streptozotocin-induced diabetes compared with control mice.

\section{U. Ahlgren}

Umeå Centre for Molecular Medicine (UCMM), Umeå University, Umeå, Sweden

\section{R. Gomis}

University of Barcelona,

Barcelona, Spain

Present address:

F. N. Pardo

Cellular and Molecular Physiology Laboratory (CMPL),

Division of Obstetrics and Gynaecology, School of Medicine,

Faculty of Medicine, Pontificia Universidad Católica de Chile,

Santiago, Chile

Present address:

J. Altirriba

Laboratory of Metabolism, Division of Endocrinology,

Diabetology and Nutrition, Department of Internal Medicine,

Faculty of Medicine, University of Geneva,

Geneva, Switzerland 
Conclusions/interpretation These data implicate RKIP1 in the regulation of pancreatic growth and beta cell expansion, thus revealing RKIP1 as a potential pharmacological target to promote beta cell regeneration.

Keywords Beta cell $\cdot$ Diabetes $\cdot$ Insulin $\cdot$ Neonatal beta cell growth $\cdot$ Proliferation $\cdot$ Raf-1 $\cdot$ RKIP

$\begin{array}{ll}\text { Abbreviations } \\ \text { ERK } & \text { Extracellular signal-regulated kinase } \\ \text { GSK3 } & \text { Glycogen synthase kinase 3 } \\ \text { IPGTT } & \text { Intraperitoneal glucose tolerance test } \\ \text { MAPK } & \text { Mitogen-activated protein kinase } \\ \text { MEK } & \text { MAP kinase kinase } \\ \text { NF-kB } & \text { Nuclear factor kB } \\ \text { P } & \text { Postnatal day } \\ \text { PDGF } & \text { Platelet-derived growth factor } \\ \text { RKIP1 } & \text { Raf-1 kinase inhibitor protein 1 } \\ \text { STZ } & \text { Streptozotocin } \\ \text { WT } & \text { Wild type }\end{array}$

\section{Introduction}

Type 2 diabetes results from pancreatic beta cell failure on a background of insulin resistance. To compensate for the increased insulin demand in insulin-resistant states, beta cells augment their insulin biosynthetic and secretory capacity in part by expanding their mass through hyperplastic and hypertrophic mechanisms [1]. However, when beta cells reach their maximum compensatory response and can no longer overcome insulin resistance, overt diabetes occurs. Hence, understanding how beta cells regulate their growth could lead to the development of therapeutic approaches to prevent and/or delay the onset of this disease.

During the last few years, our group has been investigating the actions of sodium tungstate on beta cell plasticity. We have shown that this agent can normalise blood glucose levels in streptozotocin (STZ)-treated rats and that this normalisation correlates with increased beta cell proliferation and neogenesis [2-4]. Recently, in a global transcriptome analysis, we identified the gene encoding the Raf-1 kinase inhibitor protein 1 (RKIP1) as one of the potential candidate genes involved in the ability of tungstate to modulate pancreatic beta cell mass [5].

RKIP1 is an evolutionarily conserved protein that was first recognised as a regulator of the MAPK signal transduction cascade through its interaction and inhibition of Raf-1 kinase activity [6]. However, upon phosphorylation by protein kinase C, RKIP1 can dissociate from Raf-1 and inhibit G protein-coupled receptor kinase 2 (GRK2) [7], a negative regulator of $\mathrm{G}$ protein-coupled receptors. Additionally, RKIP1 has been implicated as a negative regulator of nuclear factor $\mathrm{kB}(\mathrm{NF}-\mathrm{kB})$-signalling pathways and an activator of glycogen synthase kinase $3 \beta$ (GSK3 $\beta$ )-dependent signalling pathways $[8,9]$. Therefore, RKIP1 mediates the crosstalk between various important cellular signalling pathways and, consequently, can influence a wide array of cellular functions including cell proliferation, differentiation and apoptosis. Thus, RKIP1 has been implicated in cancer, neurological functioning and reproduction (reviewed in [10-12]).

In the pancreas, RKIP1 is present at high levels in islets, whilst its presence in exocrine cells is somewhat controversial $[13,14]$. The role of RKIP1 in beta cells is not fully understood. Because modulation of RKIP1 levels in the beta cell line HIT-T15 influences MAPK signalling and cell proliferation, it has been speculated that the presence of high amounts of RKIP1 in adult beta cells could partially explain the low proliferative capacity of these cells [13]. In line with this, overproduction of Raf-1 kinase in primary beta cells enhances cell proliferation [15]. Conversely, Raf-1 kinase inhibition results in increased beta cell apoptosis, indicating that modulation of this pathway may also contribute to beta cell survival [16]. Taken together, this in vitro evidence supports a possible role of RKIP1 in the modulation of pancreatic beta cell plasticity.

The aim of the present study was to investigate the involvement of RKIP1 in the regulation of beta cell mass using mice with a homozygous germ-line deletion of the

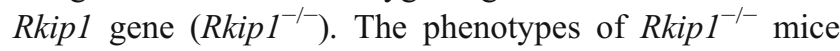
described to date include age-dependent learning and olfaction deficits and fertility defects within the males [12, 17]. Here we have characterised pancreatic morphology and glucose homeostasis in Rkipl $1^{-/}$and provide evidence of a role of RKIP1 as a negative regulator of beta cell mass under physiological (normal growth) and pathological (STZ-induced diabetes) conditions in vivo.

\section{Methods}

Mouse studies Rkip $1^{-/}$mice were generously provided by Dr Jan Klysik (Brown University, Providence, Rhode Island, USA), maintained in a C57BL/6 background and genotyped as described previously [17]. Protocols were approved by the Animal Ethics Committee of the University of Barcelona and the Principles of Laboratory Animal Care were followed. Diabetes was induced by intraperitoneal injection of $100 \mathrm{mg} / \mathrm{kg}$ STZ (Sigma-Aldrich, St Louis, MO, USA) in adult mice weighing $18-22 \mathrm{~g}$ that were fasted for $6 \mathrm{~h}$. Blood glucose levels were measured weekly until the time they were killed.

For intraperitoneal glucose tolerance tests (IPGTTs), mice fasted for $6 \mathrm{~h}$ were injected with $2 \mathrm{~g}$ glucose $/ \mathrm{kg}$ body weight. Blood samples were collected at $0,15,30,60$ and $120 \mathrm{~min}$ after injection and glycaemia was measured using a 
clinical glucometer and Accu-Chek test strips (Roche, Basel, Switzerland). Plasma was separated by centrifugation and kept at $-80^{\circ} \mathrm{C}$ for insulin determination. For insulin tolerance tests, animals were injected with $0.75 \mathrm{U}$ insulin $/ \mathrm{kg}$ body weight. Blood samples were collected at $0,15,30$ and $60 \mathrm{~min}$ and glucose levels were measured as indicated above.

Islet studies Islets were isolated from 28-day-old (P28) or 8 -week-old male mice by collagenase digestion. P28 islets were individually handpicked under a stereomicroscope after undergoing collagenase digestion, whereas adult islets were purified using a Histopaque gradient (Sigma-Aldrich) [18]. Insulin secretion was evaluated using freshly isolated islets in static incubation assays [19]. Insulin was measured by ELISA (Mercodia, Uppsala, Sweden).

Immunofluorescence and morphometric analysis Pancreases from P28 and adult mice were dissected, fixed overnight in 10\% formalin neutral buffered solution (Sigma-Aldrich) and embedded in paraffin. Sections $(4 \mu \mathrm{m})$ were deparaffinised, rehydrated and treated with citrate buffer $(10 \mathrm{mmol} / \mathrm{l} ; \mathrm{pH} 6.0)$ in a microwave oven. Primary antibodies used were: rabbit anti-RKIP (1:1,000; Calbiochem, Darmstadt, Germany), guinea pig antiinsulin (1:2,500; Dako, Glostrup, Denmark) and rat anticytokeratin 19 (DSHB, Iowa City, IA, USA). Cy2- and Cy3labelled secondary antibodies (1:200; Jackson ImmunoResearch, Newmarket, UK) were used for immunofluorescence. Hoechst 33258 (Sigma-Aldrich) was used as nuclear marker. Images were taken with a Leica DMR HC epifluorescence microscope and a Leica TCS-SL confocal microscope (Leica Microsystems, Wetzlar, Germany).

For morphometric analysis, serial sections from three different levels for each pancreas block (six sections/animal) were stained with guinea pig anti-insulin antibody as used for immunofluorescence or with mouse anti-glucagon (1:1,000; Dako) using a modified avidin-biotin-peroxidase method and counter-stained with toluidine blue [20]. Slides were analysed using Image $\mathrm{J}$ (National Institutes of Health, Bethesda, MD, USA) software.

Proliferation and apoptosis measurements For proliferation analysis, sections were stained with mouse anti-Ki67 (1:20; Dako, Denmark) as indicated above. A minimum of 3,000 insulin-positive cells per pancreas was counted in adult, P14 and P28 pancreases. For detection of apoptosis, the ApoAlert DNA fragmentation Assay Kit (Clontech, Mountain View, CA, USA) for tissue sections was used following manufacturer's instructions.

Western blot Protein extracts from whole pancreas and islets were prepared in lysis buffer $(50 \mathrm{mmol} / 1$ Tris $\mathrm{pH} 7.5$, $5 \mathrm{mmol} / \mathrm{l}$ EDTA, $150 \mathrm{mmol} / \mathrm{l} \mathrm{NaCl}, 1 \%$ (vol./vol.) Triton $\mathrm{X}-100,10 \mathrm{mmol} / \mathrm{l}$ sodium phosphate, $10 \mathrm{mmol} / \mathrm{l}$ sodium fluo- ride, $10 \mathrm{mmol} / \mathrm{l}$ sodium orthovanadate and protease inhibitors). Antibodies used for western blot were: RKIP $(1: 6,000)$, phosphorylated extracellular signal-regulated kinase 1 or 2 (ERK1/2) and total ERK1/2 (1:1,000; Cell Signaling, Beverly, MA, USA), GSK3 (1:1,000; Santa Cruz Biotechnology, Santa Cruz, CA, USA) and, as loading controls, actin (1:5,000; GE Healthcare, Hertfordshire, UK) or tubulin (1:1,000; Sigma-Aldrich).

RNA isolation and quantitative PCR analysis Total RNA was prepared from whole pancreas using RNAgents Total RNA Isolation System (Promega, Madison, WI, USA) and from isolated islets using RNeasy Micro Kit (Qiagen, Venlo, the Netherlands). RNA was treated with DNase (Qiagen), and integrity was assessed using the Agilent 2100 Bioanalyzer (Agilent Technologies, Santa Clara, CA, USA). RNA was reverse transcribed using SuperScript VILO (Invitrogen, Carlsbad, CA, USA) following the manufacturer's instructions. Real-time PCR was carried out in a LightCycler 480 System (Roche) using SYBR Green (Mesa Green, Eurogentec, Seraing, Belgium). The primer sequences used are presented in electronic supplementary material Table 1 . Expression levels were normalised to the expression of the gene encoding TATA box binding protein $(T b p)$ gene and expressed relative to the mean of the values of the same gene found in control wild-type (WT) animals, set at 1 .

Microarray analysis RNA from three WT and three Rkip $1^{-/}$ pancreases was reverse transcribed, labelled and hybridised to mouse genome 430A 2.0 arrays (Affymetrix, Santa Clara, CA, USA) according to the manufacturer's protocol. Raw data was background-adjusted, normalised and summarised by robust multi-array analysis (RMA) using the Affy package [21]. Raw and processed data were submitted to several quality controls and analysed for statistical differences as described previously [5]. Briefly, analysis was performed with the LIMMA software package available from Bioconductor (www.bioconductor.org) [22], adjusting $p$ values using Benjamini and Hochberg's method to control the false discovery rate at a value $<5 \%$ [5]. Genes were clustered and represented in a heat diagram with dChip software (http:// biosun1.harvard.edu/complab/dchip) [23]. Data have been deposited in NCBIs Gene Expression Omnibus (www.ncbi.nlm. nih.gov/geo/), accession number GSE31150.

Statistical analysis Data are expressed as mean \pm SEM and statistical significance determined by Student's $t$ test. A $p$ value $<0.05$ was considered statistically significant.

\section{Results}

RKIP1 levels in the mouse pancreas We characterised RKIP1 levels in the adult mouse pancreas by immunofluorescence 
and found that it was highly abundant in islets, where it colocalised largely, but not exclusively, with insulin (Fig. 1a). RKIP was also found in cytokeratin19+ ductal cells whereas it was not detectable in acinar cells (Fig. 1b). RKIP enrichment in islets relative to total pancreatic tissue was further confirmed by western blot (Fig. 1c). Pancreatic tissue from Rkip1 $1^{-/-}$animals was used to verify that both the staining (Fig. 1a,b) and the immunoblot band (Fig. 1c) detected in pancreases from WT mice corresponded to RKIP1.

a
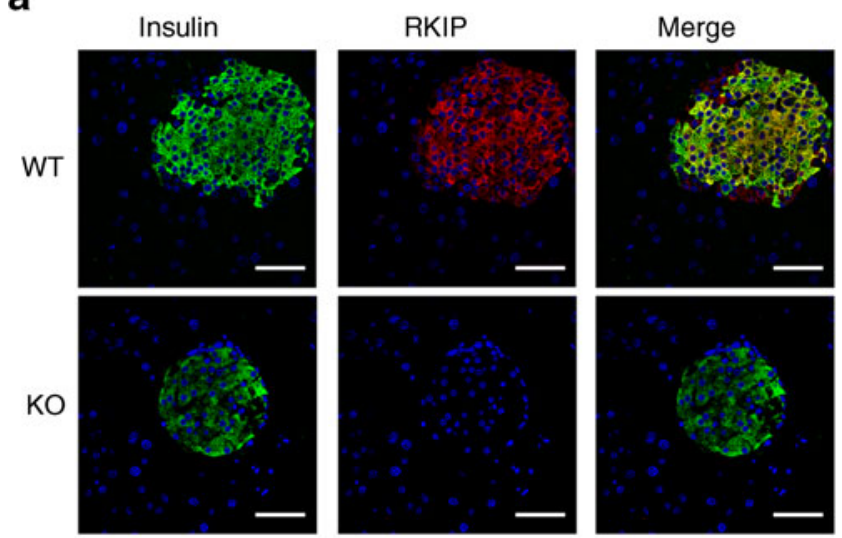

b
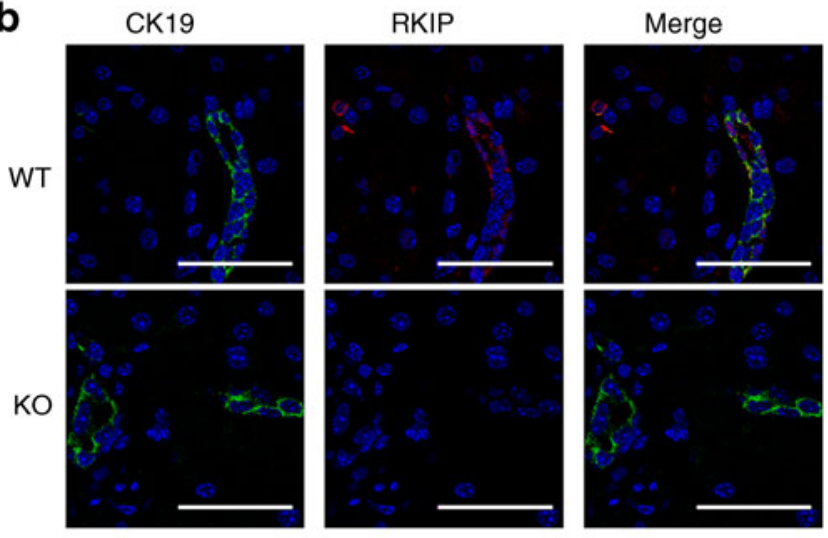

c

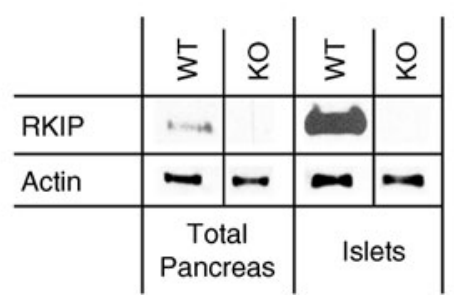

Fig. 1 Localisation of RKIP1 in the adult mouse pancreas. (a,b) Pancreases were harvested from 8-week-old male WT and Rkip1 $1^{-/}$ (KO) mice, and fixed and stained with the indicated antibodies. In (a), insulin is indicated in green, RKIP in red. In (b), ductal cell marker cytokeratin 19 is indicated in green, RKIP in red. Nuclei are marked with Hoechst 33258 and indicated in blue. Scale bars represent $50 \mu \mathrm{m}$. (c) Western blot analysis of lysates from total pancreas and isolated islets obtained from Rkipl ${ }^{-/-}$and WT mice. Actin was used as a loading control $(20 \mu \mathrm{g} / \mathrm{lane})$
Glucose homeostasis in adult Rkip $1^{-/}$animals To examine the role of RKIP1 in endocrine pancreas function, we assessed whole body glucose homeostasis in 8-week-old Rkipl $1^{-/}$animals. We found no significant differences in body weight (WT $17.8 \pm 2.3 \mathrm{~g}$, Rkipl $^{-/} 19.0 \pm 3.0 \mathrm{~g}$ ), fasting blood glucose (WT $6.8 \pm 0.4 \mathrm{mmol} / \mathrm{l}$, Rkipl $\left.^{-/-} 7.1 \pm 0.3 \mathrm{mmol} / \mathrm{l}\right)$ or fasting plasma insulin levels (WT $92.7 \pm 10.9 \mathrm{pmol} / \mathrm{l}$, Rkipl $^{--}-80.1 \pm$

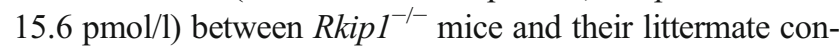
trols. However, following an intraperitoneal glucose challenge, Rkip $1^{-/}$mice displayed improved glucose tolerance (Fig. 2a,b), which correlated with increased plasma insulin 15 min after the glucose challenge (Fig. 2c). Rkipl ${ }^{-/-}$mice and WT littermates showed similar sensitivity in an insulin tolerance test (Fig. 2d). Altogether, these data indicate that Rkipl ablation results in improved glucose homeostasis and point to the pancreas as the source for this improvement.

Beta cell function and mass in Rkip $1^{-1}$ animals Since Raf1 activity is critical for insulin secretion [16, 24, 25] and insulin synthesis [25], we examined whether Rkip1 ablation affected these functions. We found that islets isolated from 8-week-old Rkip $1^{-/-}$and WT animals exhibited similar basal and glucose-induced insulin secretion and had comparable insulin contents (Fig. 3), thus ruling out a major contribution of differences in islet function to enhanced glucose homeo-

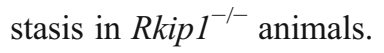

a

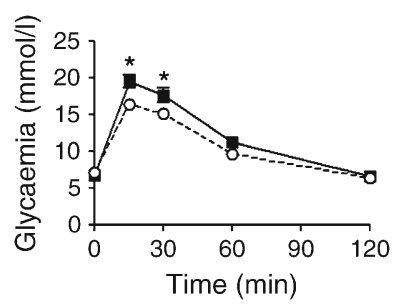

C

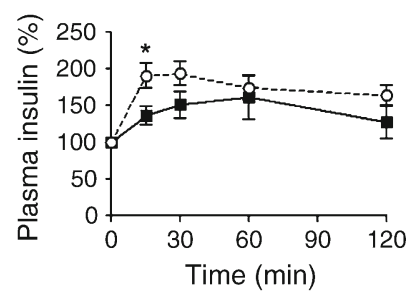

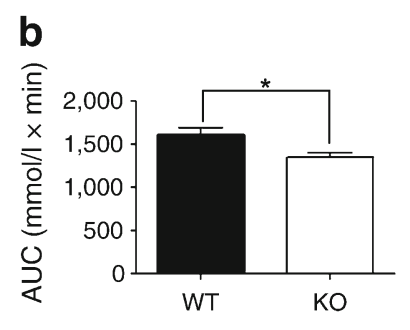

d

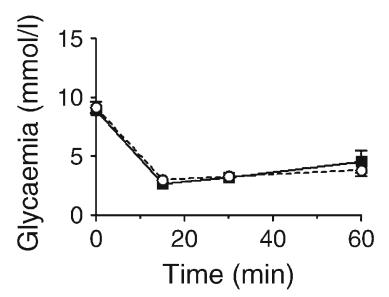

Fig. 2 Glucose tolerance and insulin sensitivity in Rkipl $^{-/-}$mice. (a) Blood glucose levels of 8-week-old Rkipl ${ }^{-/-}(\mathrm{KO}, n=8)$ and WT $(n=6)$ mice were measured at the indicated times after intraperitoneal delivery of glucose. (b) The AUC for glucose was calculated using the trapezoidal rule. (c) Plasma insulin levels after glucose administration in an IPGTT; values are expressed relative to time 0 , which is defined as $100 \%$. (d) Insulin tolerance test: blood glucose levels of 8-week-old Rkip $^{-1-}(n=3)$ and WT $(n=3)$ mice were measured at the indicated times after intraperitoneal injection with insulin. Black squares, WT animals; white circles, Rkip $1^{-/-}$animals. All results represent mean \pm SEM.* $p<0.05$ for KO vs WT 
a

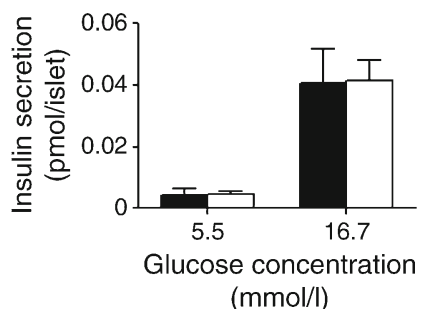

b
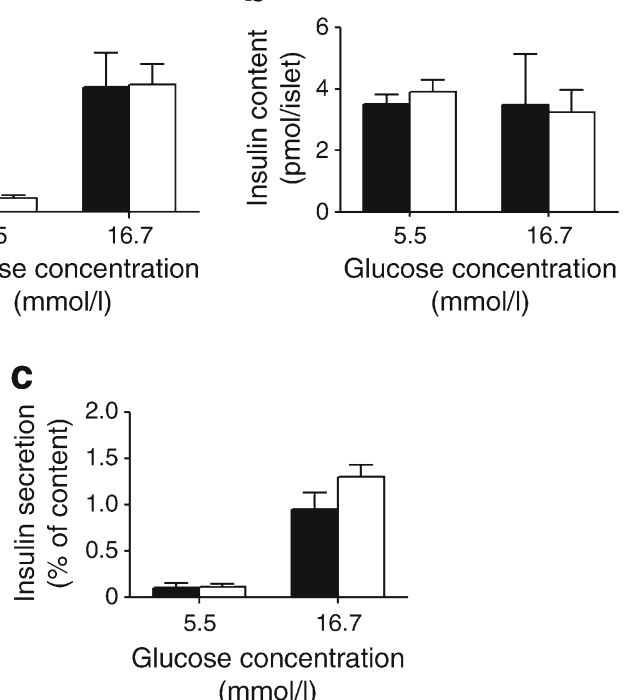

$(\mathrm{mmol} / \mathrm{l})$

Fig. 3 Glucose-induced insulin secretion in isolated islets from Rkip $^{-/-}$mice. Islets were isolated from adult WT (black bars) and Rkipl $^{-/}$(white bars) mice and insulin secretion was assayed at the indicated glucose concentrations in static incubation experiments as indicated in the Methods section. (a) Insulin secretion per islet, (b) islet insulin content, and (c) insulin secretion as percentage of total insulin content. Data are the mean \pm SEM for $n=3$ independent experiments

Alternatively, improved glucose tolerance may result from augmented beta cell mass [26]. To test this possibility, we performed morphometric analysis on fixed pancreatic tissue and found that fractional beta cell area (relative to total pancreatic area) was similar in Rkipl $^{-/-}$and WT mice (Fig. 4a). Similarly, optical projection tomography [27] failed to detect major differences in beta cell distribution throughout the whole pancreas between Rkipl $^{-/-}$and WT animals (ESM Fig. 1). However, Rkip $1^{-1-}$ animals

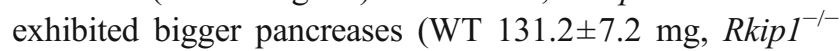
$185.1 \pm 7.3 \mathrm{mg} ; p<0.001$ ), which resulted in a nearly twofold

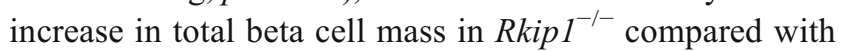
WT mice (Fig. 4b).

No differences in islet cell composition or beta cell volume were apparent between WT and Rkip ${ }^{-/-}$mice (Fig. 4c,d). Yet, Rkip ${ }^{-/-}$pancreases displayed significant alterations in islet size distribution, with more small islets and fewer medium and large islets in kipl $^{-/-}$compared with WT animals (Fig. 4e).

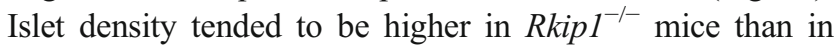
controls (Fig. 4f), which could explain why, despite exhibiting a larger proportion of small islets, the relative beta cell area remained similar in Rkip1 ${ }^{-/-}$mice to that in WT animals. The percentage of islets located near ducts $(<5 \mu \mathrm{m})$ was significantly increased in Rkip ${ }^{-/-}$animals (Fig. 4g).

Taken together, these data indicate that an augmented beta cell mass:body weight ratio, rather than alterations in beta cell functionality, is responsible for the improvement in whole body glucose control in RkipI $1^{-/}$mice [26]. Furthermore, a

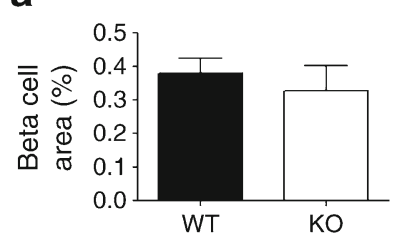

b

c

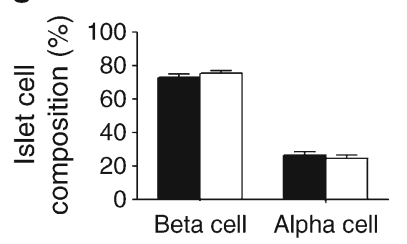

d

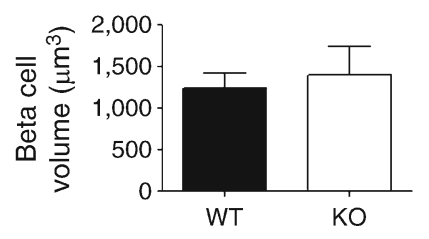

f
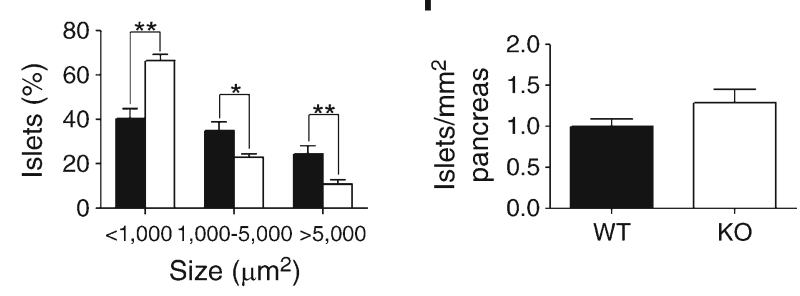

g

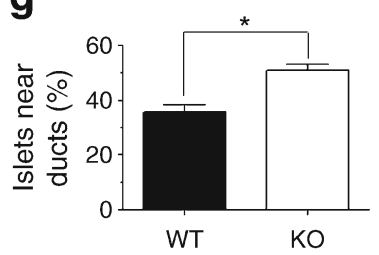

Fig. 4 Characterisation of the pancreatic beta cell compartment in adult Rkip $^{-1-}$ mice. Morphometric analysis of fixed pancreas from adult Rkip $^{-/-}(\mathrm{KO}, n=8)$ and WT $(n=8)$ mice. (a) Beta cell area calculated as insulin-positive islet area (in $\mu \mathrm{m}^{2}$ ) as a percentage of the total pancreatic area (in $\mu \mathrm{m}^{2}$ ), (b) total beta cell mass, (c) islet cell composition, (d) beta cell volume, (e) islet size distribution, (f) islet density and (g) percentage of islets located at $<5 \mu \mathrm{m}$ from ducts. WT animals are shown in black bars and Rkip $1^{-/}$animals in white bars. Data are the mean \pm SEM. ${ }^{*} p<0.05$ and $* * p<0.01 \mathrm{KO}$ vs WT

while the relevance of altered islet size distribution and location in the control of glucose homeostasis remains unclear, our findings unravel additional potential effects of RKIP1 on islet morphogenesis.

Beta cell proliferation in Rkip1 $1^{--}$animals We next examined the mechanisms underlying the increased beta cell mass in Rkip $^{-/-}$mice. Since RKIP1 has been shown to inhibit beta cell proliferation in vitro [13], we investigated whether the augmented beta cell mass in Rkip $1^{-/-}$animals was due to increased proliferation. Co-immunostaining for insulin and Ki67 in pancreases of adult animals failed to detect any significant difference in cell proliferation rates between Rkip $1^{-/-}$mice and control littermates (Fig. 5a). We then postulated that beta cell mass might have been augmented 

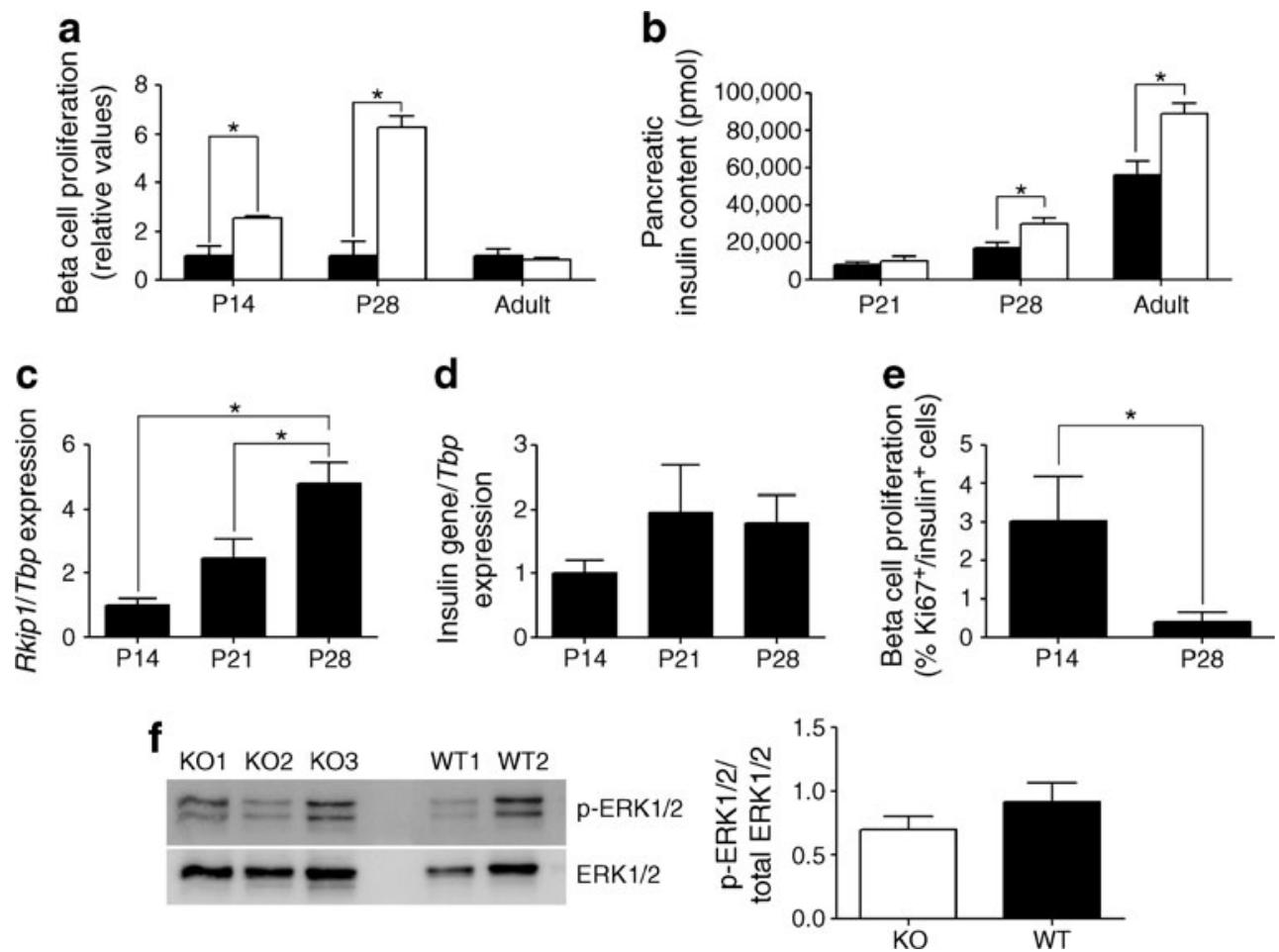

Fig. 5 Beta cell proliferation in Rkip $1^{-/-}$mice. (a) Relative levels of proliferating beta cells $\left(\mathrm{Ki}_{67}{ }^{+} /\right.$insulin $\left.^{+}\right)$in P14, P28 and 8-week-old Rkip $^{-/-}$and WT pancreases ( $n=3-6$ animals per group). For comparison purposes, data are expressed relative to levels found in agematched WT mice, set at 1. (b) Total pancreatic insulin content was measured by ELISA in P21, P28 and adult Rkip1 $1^{-/-}$mice $(n=5-6)$ and WT (n=5-6) littermates. (c,d) quantitative RT-PCR for Rkipl and Ins $1+2$ mRNAs in pancreases harvested from P14, P21 and P28 WT mice $(n=3-4)$. Values are expressed relative to Tbp. (e) Percentage of

during young postnatal life, when the greatest expansion of beta cells takes place [28]. Consistent with this idea, we found that Rkip1 $1^{-/-}$animals exhibited a higher number of proliferating beta cells at P14 and P28 than controls of the same age (Fig. 5a). Total pancreatic insulin content was also significantly increased in P28 Rkipl $1^{-/}$mice relative to age-matched controls, and this difference was maintained in adult animals (Fig. 5b). Hence, RKIP1 appears to regulate beta cell proliferation in vivo specifically during the early postnatal period.

Our previous results suggested that RKIP1 might be involved in restricting beta cell expansion specifically around the lactation-weaning transition (P21-P28). To further support this possibility, we assessed whether changes in endogenous Rkipl expression correlated with inhibition of beta cell proliferation during this transition in control mice. We observed a stepwise rise in the abundance of pancreatic Rkipl mRNA, accompanied by a significant drop in beta cell proliferation rates between P14 and P28 (Fig. 5c,e). Remarkably, increased Rkipl expression was not correlated with an increase in insulin (Ins 1 and Ins2) gene expression (Fig. 5d). Thus, these findings proliferating beta cells $\left(\mathrm{Ki}_{6} 7^{+} /\right.$insulin $\left.^{+}\right)$in pancreases from control mice at P14 and P28 ( $n=3-6$ animals per group). (f) Representative immunoblot for phosphorylated ERK1/2 (p-ERK1/2) and total ERK1/2 in isolated islets from P28 Rkipl ${ }^{-/-}(\mathrm{KO})$ and WT control littermates. Bands were quantified by densitometry and values expressed as the ratio of $\mathrm{p}$-ERK1/2 relative to total ERK1/2 protein for $n=6$ per genotype. Black bars, WT animals; white bars, Rkip $1^{-/-}$animals. All bars represent the mean \pm SEM $* p<0.05$ between indicated groups

support the notion that activation of Rkipl expression could participate in restricting beta cell expansion at the end of lactation.

To elucidate the molecular basis for the enhanced beta cell proliferation in P28 Rkipl ${ }^{-/}$animals we examined the phosphorylation status of the MAPKs ERK1/2, which are the canonical targets of the Raf-1/MAP kinase kinase (MEK) pathway. However, we found no significant changes in basal ERK1/2 phosphorylation in islets freshly isolated from Rkip $1^{-/}$mice relative to those from controls (Fig. 5f). RKIP1 also enhances GSK3 $\beta$-dependent signalling through stabilisation of this kinase [9]. GSK3 $\beta$ is involved in $\beta$-catenin turnover and $\mathrm{WNT} / \beta$-catenin signalling has been shown to regulate neonatal beta cell expansion [29]. Accordingly, GSK3 $\beta$ deficiency results in expanded beta cell mass [30]. These findings prompted us to examine whether the absence of RKIP1 affected GSK3 $\beta$ protein accumulation. However, we found no differences in GSK $3 \beta$ protein or Axin 2 mRNA expression (an endogenous indicator of $\mathrm{WNT} / \beta$-catenin signalling) between P28 Rkipl ${ }^{-/-}$and WT islets (ESM Fig. 2). Thus, although alterations in a small subset of beta cells or transient modulation of these pathways cannot be ruled out, 
these results indicate that neither MAPK nor GSK3 $\beta$ are constitutively changed in Rkipl $^{-/-}$islets.

Pancreatic growth in Rkip1 $1^{-/}$animals The finding that Rkipl ablation affected the pancreatic endocrine and exocrine compartments in a similar manner indicates that RKIP1 might also regulate exocrine cell proliferation. This result was unexpected because of the absence of RKIP1 in adult acinar cells (Fig. 1). To investigate this issue further, we determined the time at which the increase in pancreas size was first evident in $\mathrm{KO}$ mice. Pancreas weights (corrected for body weights) were similar between $\mathrm{KO}$ and control littermates until P28 (Fig. 6a), at which time Rkip $^{-1-}$ mice showed a higher pancreas weight, thus indicating that this phenotype is not determined during embryogenesis but, as for beta cell proliferation, during young postnatal life, around the lactation-weaning transition (P21-P28). Consistent with enhanced pancreatic growth, total pancreas (endocrine+exocrine) proliferation rates measured by Ki67 staining were dramatically increased in

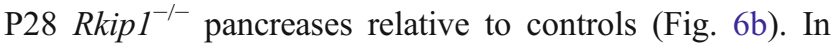
contrast, apoptotic rates as measured by TUNEL assay were negligible and similar between P28 Rkipl $^{-1-}$ mice and controls (Fig. 6c). In view of these results, we wondered whether the pattern of RKIP1 distribution in the young postnatal pancreas might differ from that of the adult organ. We found that, as in the adult, RKIP1 was preferentially located in islet and ductal cells at P21 and P28 (Fig. 6d and data not shown). However, we also noticed distinctive nuclear staining in many acinar cells at these stages (Fig. 6d), thus indicating that ablation of the Rkipl gene in postnatal acinar cells might be involved in enhanced exocrine cell proliferation in a cell autonomous manner in Rkip $^{-/-}$mice.

To elucidate the pathways responsible for increased pancreatic growth in Rip $^{-/-}$mice, we performed whole genome expression profiling using pancreases from P28 $\mathrm{Rkip}^{-/-}$and WT animals. Gene ontology analysis identified the cell cycle as the most significantly represented pathway among RKIP1regulated genes (ESM Fig. 3). Among the genes most highly upregulated in P28 Ripl $^{-1-}$ pancreases relative to controls, we identified those encoding the four members of the superfamily of C-type lectins known as regenerating islet-derived 3: Reg3a, Pap (also known as Reg3b), Ingap (also known as $\operatorname{Reg} 3 d$ ) and $\operatorname{Reg} 3 g$. Interestingly, Reg3 genes have been shown to function as stress response genes, antiapoptotic and growth factors in the digestive system and to specifically promote beta cell proliferation in the pancreas [31]. We confirmed these data by quantitative RT-PCR and found that
Fig. 6 Effects of Rkip1 deletion on pancreatic growth. (a) Relative pancreatic weight (corrected for body weight) at P1, P7, P14, P21, P28 and in 8-week-old Rkip1 ${ }^{-1-}(n=10)$ and WT $(n=10)$ mice. (b) Percentage of proliferating pancreatic cells $\left(\mathrm{Ki} 67^{+}\right)$in pancreases from P28 Rkip1 ${ }^{-/}$ $(\mathrm{KO})$ and WT mice $(n=6)$ and a representative image of a P28 Rkip $1^{-/}$pancreas showing immunostaining for Ki67 (green) in non-insulin and insulin-positive (red) cells Nuclei are marked with Hoechst 33258 (blue). (c) Percentage of apoptotic cells in pancreases from P28 Rkip $1^{-/}$(KO) and WT mice $(n=6)$. (d) Representative images showing immunostaining for RKIP (red), cytokeratin 19 (green) and insulin (blue) on pancreatic sections from P21 WT and Rkipl $^{-/-}$(KO) animals. Black bars, WT animals; white bars Rkip $^{-/-}$animals. All bars represent the mean \pm SEM. ${ }^{*} p<0.05$ between indicated groups. Scale bars represent $50 \mu \mathrm{m}$ a
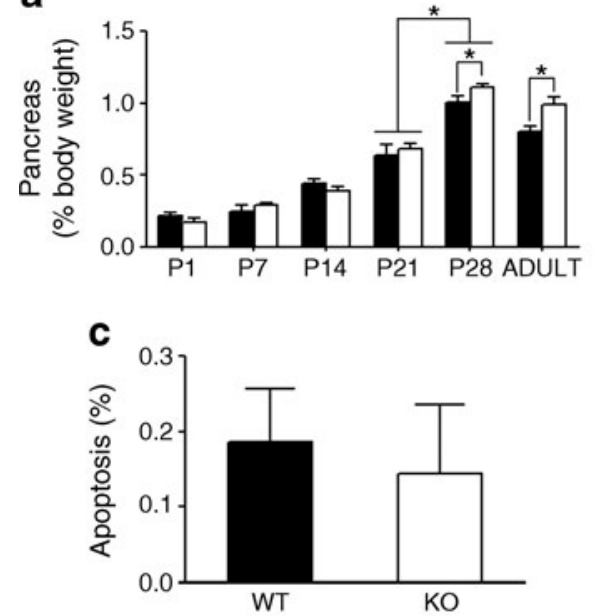

d

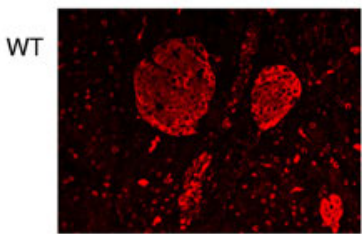

$\mathrm{KO}$

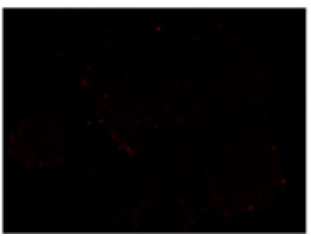

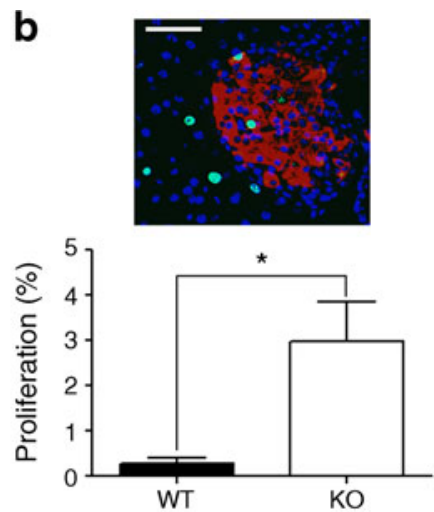


Reg3 overexpression was only seen after weaning (ESM Fig. 3). However, Reg3 mRNAs were not overexpressed in isolated islets from P28 Rkip $^{-/-}$relative to WT animals (MPJ, RG unpublished observations), pointing to the exocrine pancreas as being responsible for Reg3 gene activation in Rkip $^{-1-}$ animals.

Taken together, these data reveal RKIP1 as a negative regulator of postnatal pancreatic cell proliferation. Since cell cycle genes are mainly regulated at the post-transcriptional level, the molecular signature of Rkipl ablation and its significance in pancreatic growth will need to be further explored through the identification of changes in cell cycle protein production in $R k i p 1^{-/-}$pancreases.

Rkip1 deletion ameliorates STZ-induced diabetes Our results thus far have demonstrated that Rkipl deletion affects beta cell proliferation in early postnatal life. To determine whether RKIP1 might be a potential target to trigger beta cell mass recovery in diabetes, adult mice were rendered diabetic by STZ injection. At 7 days post-injection, both Rkip $^{-/-}$and WT animals treated with STZ became hyperglycaemic (WT/STZ $14.1 \pm 1.4$ vs WT/control $7.7 \pm$

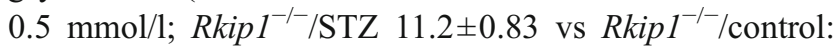
$8.7 \pm 0.4 \mathrm{mmol} / \mathrm{l}$ ) (Fig. $7 \mathrm{a}$ ) and exhibited $>80 \%$ depletion of Ins 2 mRNA levels (Fig. 7b). The onset of hyperglycaemia and the marked loss of Ins 2 mRNA indicate that beta cells are efficiently eliminated by STZ in both Rkip $^{-/-}$and WT groups. One week later, Rkip $1^{-/-}$mice exhibited near normalisation of blood glucose levels, whereas WT animals became severely hyperglycaemic and remained diabetic until the end of the study (Fig. 7a). The recovery of Rkip $1^{-1-}$ mice correlated with increased Ins 2 mRNA levels at 14 days after the injection (Fig. 7b). At 28 days postinjection, STZ-treated Rkip $^{-/-}$mice had normalised plasma insulin levels and had an increased pancreatic insulin content (Fig. 7c,d) compared with STZ-treated control mice, pointing to the partial rescue of beta cell mass in Rkipl ${ }^{-/}$ animals and implying that beta cell regeneration participates in the restoration of normoglycaemia in these animals. In sum, these findings indicate that RKIP1 can also regulate beta cell mass in an experimental model of diabetes.

\section{Discussion}

How beta cell mass is regulated is a critical issue for understanding the pathophysiology of diabetes, which is characterised by a complete (type 1) or relative (type 2) deficiency in beta cell number. During mouse embryogenesis, new beta cells arise through the proliferation and differentiation of a population of progenitor cells. During postnatal development, between birth and weaning, there's an additional and massive increase in beta cell mass that mainly involves

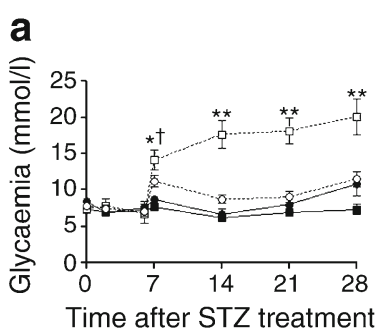

(days)

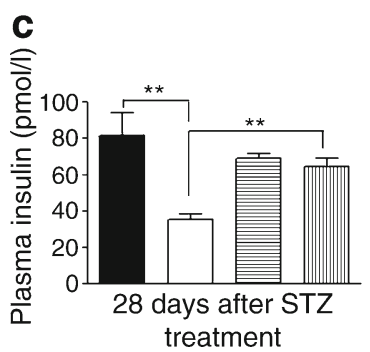

b

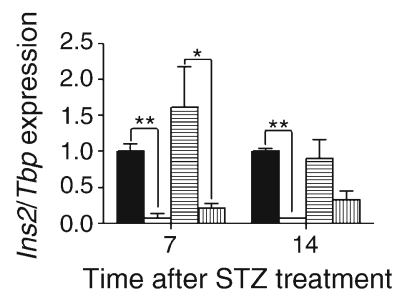

(days)

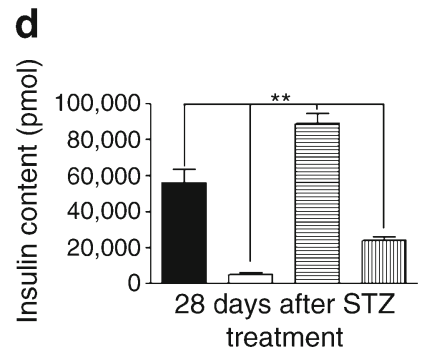

Fig. 7 Reversal of STZ-induced diabetes in kkip $^{-/-}$mice. (a) Glucose plasma levels after STZ (white symbols, dotted line) or vehicle (control, black symbols, solid line) injection in WT (squares) and Ripl $^{-/-}$(KO, circles) mice ( $n=7$ for each group); ${ }^{\dagger} p<0.05, \mathrm{KO}$ control vs KO STZ; ${ }^{*} p<0.05,{ }^{* *} p<0.01$ WT control vs WT STZ. (b) Ins 2 mRNA expression in whole pancreas from WT and Rkip $1^{-1-}$ mice 7-days and 14-days post STZ or saline injection. For each time point, Ins 2 mRNA expression of WT mice injected with vehicle (WT control) is set at $1(n=5)$. (c) Plasma insulin levels of WT and kip $^{-/-}$mice 28 days after injection with STZ or vehicle $(n=5)$. (d) Pancreatic insulin content of WT and Rkip ${ }^{-/-}$mice 28 days after injection with STZ or vehicle $(n=4)$. Black bars, WT animals treated with vehicle; white bars, WT animals treated with STZ; horizontal lines, KO control, vertical lines, KO STZ. All data are the mean \pm SEM. ${ }^{*} p<0.05,{ }^{*} p<0.01$ between indicated conditions

replication from pre-existing beta cells, even though neogenesis is maintained at least during the first week of life. By the time of weaning, beta cell proliferation decreases and is maintained relatively low throughout adulthood (for reviews, see [26, 32]). In this study we aimed to investigate the physiological role of RKIP1 in the regulation of beta cell mass. Our results indicate that RKIP1 participates in the control of pancreatic growth, particularly at the time of weaning. In addition, we demonstrate that the absence of RKIP1 confers significant protection against STZ-induced diabetes, thus indicating that this protein may negatively influence beta cell regeneration after injury in the adult pancreas.

Rkip ${ }^{-/-}$animals exhibit increased beta cell mass relative to body weight and this augmentation, in the absence of changes in insulin sensitivity, is the likely cause for their improved glucose tolerance. Indeed, beta cell mass expansion and body weight follow a linear correlation throughout life [28], and alterations in this correlation are known to affect glucose homeostasis [26]. A recent study showed that beta cell-specific deletion of Raf-1 kinase results in the downregulation of Ins 2 gene transcription and, consequently, in blunted insulin secretion [25]. In contrast, we have found 
that the insulin content, insulin gene expression and glucose-induced insulin secretion of kip $^{-/-}$islets is similar to that of WT islets (Fig. 3 and data not shown), arguing against a major involvement of altered islet function in enhanced whole body glucose control in Rkip $^{-1-}$ mutant mice. Yet, it is conceivable that, in adult islets under normal physiological conditions, Raf-1 kinase is already maximally stimulated, so that elimination of RKIP1 has no measurable effects on Raf-1 kinase-dependent processes.

In accordance with prior in vitro experiments [13], we have observed that RKIP1 inhibits beta cell proliferation in vivo. Remarkably, the inhibitory effect of RKIP1 is only evident during early postnatal stages when the beta cell proliferative capacity is maximal. This age-dependent loss of the effects of RKIP1 may simply reflect changes in the repertoire of signalling factors available to the beta cell at any given moment in time. Alternatively, changes in the responsiveness of beta cells to specific signalling molecules, rather than their availability, may underlie the age-dependent effects of Rkipl ablation. In line with this, Chen et al [33] have recently linked agetriggered loss of beta cell expression of the platelet-derived growth factor (PDGF) receptor to reduced beta cell proliferation. Hence, it is tempting to speculate that the proliferative response to Rkipl ablation will be lost in parallel with the loss of the ability of the beta cell to respond to specific factors.

Genetic mouse models and in vitro studies have helped unravel the identity of growth factors that stimulate beta cell replication, but their role under normal physiological conditions remains uncertain. Among the pathways implicated, many transduce signals through the Raf-1/MEK/ERK cascade, including insulin, insulin-growth factor, hepatic growth factor, epidermal growth factor and PDGF [34-36], and thus could potentially be targeted by RKIP1. Yet, our data indicate that constitutive ERK1/2 activation may not be linked to enhanced beta cell proliferation in Rkipl mutants. In a similar way, deletion of Raf-1 does not result in decreased ERK1/2 phosphorylation in pancreatic islets, indicating that other Raf1-dependent pathways may be operative in beta cells [25]. Additionally, RKIP1 may function through Raf-1 independent mechanisms involving NF-kB [8], GSK3 $\beta$ [9] or G protein-coupled receptors [11]. Apoptotic rates are comparable between Rkip $^{-/-}$and WT P28 pancreases, implying that NF-кB-dependent survival pathways are not affected by Rkip1 deletion. On the other hand, RKIP1 deficiency does not alter levels of GSK3 $\beta$ in islets, as reported in other tissues [9], ruling out this possible mechanism. Future work should be aimed at delineating the signal cascades regulated by RKIP1 and their connection to beta cell mitogens during physiological beta cell growth.

This study reveals that RKIP1 is not only involved in restricting beta cell proliferation but it may also inhibit postnatal pancreatic growth. Gene expression profiling has identified the Reg3 gene family as potentially involved in the stimulatory effect of RKIP1 ablation on pancreatic growth at P28. Interestingly, the Reg3 genes have been previously associated to pancreatic regeneration and islet cell neogenesis [37-41]. Further supporting a role for Reg3 genes in pancreatic postnatal development, a recent study has described the upregulation of Reg3 genes in the mouse pancreas between P7 and P30 followed by their subsequent downregulation in adulthood [42]. In this scenario, our data suggest that RKIP1 may participate in restricting Reg3 gene expression after weaning and, in this way, control pancreatic growth. The question of whether exocrine Reg3 gene activation might contribute to enhanced beta cell proliferation in Rip $^{-/-}$mice remains unresolved. Double Reg3 and Rkipl mutants and beta cell-specific Rkip1 ${ }^{-/}$mice will be necessary to properly address this issue.

In summary, in this paper we show that RKIP1 inactivation results in enhanced beta cell and pancreatic growth in postnatal stages, leading to an increment in beta cell mass and improved glucose tolerance in adult animals. Furthermore, Rkip1 deletion confers recovery to STZ-induced hyperglycaemia in adult animals. These results have therapeutic implications for the development of novel strategies to trigger beta cell mass expansion in diabetes.

Acknowledgements The authors thank A. Eriksson (Umeå University) for the optical projection tomography analyses.

Funding This work has been supported by grants from: Spanish Ministerio de Ciencia e Innovación (SAF2010-19527 to R. Gomis and BFU2008-02299/BMC to R. Gasa), Generalitat de Catalunya (2009 SGR 1426 to R. Gomis), Fondecyt no. 1090694 (Chile) and Fundación Marcelino Botin (project 08/351) and the European Community's Seventh Framework Programme (FP7/2007-2013) under grant agreement no. PIAP-GA-2008-218130 (to R. Gomis). Predoctoral fellowships were provided by Fundación Marcelino Botin (F. N. Pardo), Conicyt-PhD (F. N. Pardo), IDIBAPS (J. Altirriba) and Spanish Ministerio de Economía y Competitividad (M. Pradas-Juni).

Duality of interest The authors declare that there is no duality of interest associated with this manuscript.

Contribution statement FNP designed, performed the study, analysed and interpreted data and drafted the manuscript. JA, MPJ, AG and UA made substantial contributions to the acquisition, analysis and interpretation of data and critically revised the manuscript. AB, JCS and AJY contributed to data analysis and interpretation and critically revised the manuscript. RGo and RGa designed the study, supervised the project, interpreted data and wrote the manuscript. All authors have approved the final version of the manuscript.

\section{References}

1. Ballian N, Hu M, Liu SH, Brunicardi FC (2007) Proliferation, hyperplasia, neogenesis, and neoplasia in the islets of Langerhans. Pancreas 35:199-206

2. Barbera A, Fernandez-Alvarez J, Truc A, Gomis R, Guinovart JJ (1997) Effects of tungstate in neonatally streptozotocin-induced diabetic rats: mechanism leading to normalization of glycaemia. Diabetologia 40:143-149 
3. Barbera A, Gomis RR, Prats N et al (2001) Tungstate is an effective antidiabetic agent in streptozotocin-induced diabetic rats: a long-term study. Diabetologia 44:507-513

4. Fernandez-Alvarez J, Barbera A, Nadal B et al (2004) Stable and functional regeneration of pancreatic beta-cell population in nSTZrats treated with tungstate. Diabetologia 47:470-477

5. Altirriba J, Barbera A, del Zotto H et al (2009) Molecular mechanisms of tungstate-induced pancreatic plasticity: a transcriptomics approach. BMC Genomics 10:406

6. Yeung K, Seitz T, Li S et al (1999) Suppression of Raf-1 kinase activity and MAP kinase signalling by RKIP. Nature 401:173-177

7. Lorenz K, Lohse MJ, Quitterer U (2003) Protein kinase C switches the Raf kinase inhibitor from Raf-1 to GRK-2. Nature 426:574-579

8. Yeung KC, Rose DW, Dhillon AS et al (2001) Raf kinase inhibitor protein interacts with NF- $\mathrm{KB}$-inducing kinase and TAK1 and inhibits NF-KB activation. Mol Cell Biol 21:7207-7217

9. Al Mulla F, Bitar MS, Al-Maghrebi M et al (2011) Raf kinase inhibitor protein RKIP enhances signaling by glycogen synthase kinase-3 $\beta$. Cancer Res 71:1334-1343

10. Keller ET, Fu Z, Brennan M (2004) The role of Raf kinase inhibitor protein (RKIP) in health and disease. Biochem Pharmacol 68:1049-1053

11. Keller ET, Fu Z, Brennan M (2005) The biology of a prostate cancer metastasis suppressor protein: Raf kinase inhibitor protein. J Cell Biochem 94:273-278

12. Klysik J, Theroux SJ, Sedivy JM, Moffit JS, Boekelheide K (2008) Signaling crossroads: the function of Raf kinase inhibitory protein in cancer, the central nervous system and reproduction. Cell Signal 20:1-9

13. Zhang L, Fu Z, Binkley C et al (2004) Raf kinase inhibitory protein inhibits beta-cell proliferation. Surgery 136:708-715

14. Kim HS, Kim GY, Lim SJ, Kim YW (2010) Loss of Raf-1 kinase inhibitory protein in pancreatic ductal adenocarcinoma. Pathology 42:655-660

15. Beith JL, Alejandro EU, Johnson JD (2008) Insulin stimulates primary beta-cell proliferation via Raf-1 kinase. Endocrinology 149:2251-2260

16. Alejandro EU, Johnson JD (2008) Inhibition of Raf-1 alters multiple downstream pathways to induce pancreatic beta-cell apoptosis. J Biol Chem 283:2407-2417

17. Theroux S, Pereira M, Casten KS et al (2007) Raf kinase inhibitory protein knockout mice: expression in the brain and olfaction deficit. Brain Res Bull 71:559-567

18. Casas S, Novials A, Reimann F, Gomis R, Gribble FM (2008) Calcium elevation in mouse pancreatic beta cells evoked by extracellular human islet amyloid polypeptide involves activation of the mechanosensitive ion channel TRPV4. Diabetologia $51: 2252-2262$

19. Altirriba J, Gasa R, Casas $S$ et al (2010) The role of transmembrane protein 27 (TMEM27) in islet physiology and its potential use as a beta cell mass biomarker. Diabetologia 53:1406-1414

20. Palau N, Rebuffat SA, Altirriba J et al (2012) Role of IGFBP-3 in the regulation of $\beta$-cell mass during obesity: adipose tissue $/ \beta$-cell cross talk. Endocrinology 153:177-187

21. Gautier L, Cope L, Bolstad BM, Irizarry RA (2004) Affy—analysis of Affymetrix GeneChip data at the probe level. Bioinformatics 20:307-315

22. Gentleman RC, Carey VJ, Bates DM et al (2004) Bioconductor: open software development for computational biology and bioinformatics. Genome Biol 5:R80
23. Li C, Wong WH (2001) Model-based analysis of oligonucleotide arrays: expression index computation and outlier detection. Proc Natl Acad Sci U S A 98:31-36

24. Kowluru A, Veluthakal R, Rhodes CJ, Kamath V, Syed I, Koch BJ (2010) Protein farnesylation-dependent Raf/extracellular signalrelated kinase signaling links to cytoskeletal remodeling to facilitate glucose-induced insulin secretion in pancreatic beta-cells. Diabetes 59:967-977

25. Alejandro EU, Lim GE, Mehran AE et al (2011) Pancreatic $\beta$-cell Raf-1 is required for glucose tolerance, insulin secretion, and insulin 2 transcription. FASEB J 25:3884-3895

26. Bouwens L, Rooman I (2005) Regulation of pancreatic beta-cell mass. Physiol Rev 85:1255-1270

27. Alanentalo T, Asayesh A, Morrison H et al (2007) Tomographic molecular imaging and 3D quantification within adult mouse organs. Nat Methods 4:31-33

28. Montanya E, Nacher V, Biarnes M, Soler J (2000) Linear correlation between beta-cell mass and body weight throughout the lifespan in Lewis rats: role of beta-cell hyperplasia and hypertrophy. Diabetes 49:1341-1346

29. Rulifson IK, Karnik SK, Heiser PW (2007) Wnt signalling regulates pancreatic beta cell proliferation. Proc Natl Acad Sci USA 104:6247-6252

30. Liu K, Tanabe D, Baronnier et al (2010) Conditional ablation of Gsk-3 $\beta$ in islet beta cells results in expanded mass and resistance to fat feeding-induced diabetes in mice. Diabetologia 53:2600 2610

31. Zhang YW, Ding LS, Lai MD (2003) Reg gene family and human disease. World J Gastroenterol 9:2635-2641

32. Bonner-Weir S, Sharma A (2002) Pancreatic stem cells. J Pathol 197:519-526

33. Chen H, Gu X, Liu Y et al (2011) PDGF signalling controls agedependent proliferation in pancreatic $\beta$-cells. Nature 478:349-355

34. Yesil P, Lammert E (2008) Islet dynamics: a glimpse at beta cell proliferation. Histol Histopathol 23:883-895

35. Heit JJ, Karnik SK, Kim SK (2006) Intrinsic regulators of pancreatic beta-cell proliferation. Annu Rev Cell Dev Biol 22:311-338

36. Cozar-Castellano I, Fiaschi-Taesch N, Bigatel T (2006) Molecular control of cell cycle progression in the pancreatic $\beta$-cell. Endocr Rev 27:356-370

37. Lu Y, Ponton A, Okamoto H et al (2006) Activation of the Reg family genes by pancreatic-specific IGF-I gene deficiency and after streptozotocin-induced diabetes in mouse pancreas. Am J Physiol Endocrinol Metab 291:E50-E58

38. Rosenberg L, Lipsett M, Yoon JW et al (2004) A pentadecapeptide fragment of islet neogenesis-associated protein increases beta-cell mass and reverses diabetes in C57BL/6J mice. Ann Surg 240:875884

39. Choi JH, Lee MY, Kim Y et al (2010) Isolation of genes involved in pancreas regeneration by subtractive hybridization. Biol Chem 391:1019-1029

40. Paula FM, Barbosa HC, Carneiro EM et al (2010) Requirement of NF-kappaB signalling pathway for modulation of the cholinergic muscarinic $\mathrm{M}_{3}$ receptor expression by INGAP-PP in insulinproducing cells. Eur J Pharmacol 642:37-46

41. Rafaeloff R, Pittenger GL, Barlow SW et al (1997) Cloning and sequencing of the pancreatic islet neogenesis associated protein (INGAP) gene and its expression in islet neogenesis in hamsters. J Clin Invest 99:2100-2109

42. Wang Y, Jacovetti C, Li B et al (2011) Coordinated age-dependent and pancreatic-specific expression of mouse Reg2, Reg3 $\alpha$, and Reg3 $\beta$ genes. Growth Factors 29:72-81 